Revue d'histoire de l'enfance « irrégulière »

Le Temps de l'histoire

19 | 2017

Abandon d'enfants et parents abandonneurs, $\mathrm{XIX}{ }^{\mathrm{e}}-\mathrm{XX} \mathrm{I}^{\mathrm{e}}$ siècles

\title{
Le couvent à sabots. Témoignage autobiographique
}

\section{Claire Dumas}

\section{(2) OpenEdition \\ Journals}

Édition électronique

URL : https://journals.openedition.org/rhei/4091

DOI : 10.4000/rhei.4091

ISSN : $1777-540 \mathrm{X}$

Éditeur

Presses universitaires de Rennes

\section{Édition imprimée}

Date de publication : 22 novembre 2017

Pagination : 205-210

ISBN : 978-2-7535-6467-1

ISSN : $1287-2431$

Référence électronique

Claire Dumas, "Le couvent à sabots. Témoignage autobiographique », Revue d'histoire de l'enfance " irrégulière » [En ligne], 19 | 2017, mis en ligne le 22 novembre 2017, consulté le 09 septembre 2021.

URL : http://journals.openedition.org/rhei/4091 ; DOI : https://doi.org/10.4000/rhei.4091 
(212 jours en quatre ans) et se conclut par son transfert avec quatre de ses camarades à la colonie correctionnelle d'Eysses, dite « Eysses la maudite ». Une fois encore des recherches minutieuses permettent de percevoir le sort réservé aux colons récalcitrants, et la violence de cette institution. Finalement, le jeune homme est libéré dans sa vingtième année et la seconde partie de l'ouvrage retrace ses quelques mois de liberté, son arrestation suite à un cambriolage avec violences, sa condamnation à dix ans de travaux forcés et sa longue agonie au bagne de Cayenne, où il meurt assassiné par un autre détenu en 1911. La conclusion offre des réflexions sur la question de l'enfermement aujourd'hui, et l'ouvrage se clôt sur des annexes riches: rapports d'incidents à Bologne, reconstitution brève du parcours des camarades de Milot, témoignages de bagnards...

Dans une même perspective que le livre novateur de Béatrice Koeppel écrit à partir du dossier de Marguerite B. ${ }^{3}$, le collectif « L'Escapade » propose ici une démarche originale et réussie. L'étude d'une trajectoire, la fouille méticuleuse d'une multitude d'archives, la plume résolument politique et vivante, permettent d'approcher de près ces vingt-six années d'existence, la destinée tragique d'un jeune apache. Un anonyme dont aucunes traces directes (écrits, images) n'ont été retrouvées mais qui nous paraît pourtant si proche une fois le livre terminé. Ce manifeste contre l'enfermement est également un véritable travail d'historien ${ }^{4}$ et une belle introduction à la justice des enfants de la Belle Époque.

Véronique Blanchard

\section{Le couvent à sabots. Témoignage autobiographique \\ Christiane Pécriaux \\ 2005}

Nantes, Éditions Amalthée, 374 p., ISBN 9782350272605.

Comme l'indique le sous-titre du livre: "témoignage autobiographique ", il s'agit du propre parcours de l'auteure, Christiane Pécriaux, infirmière de métier, qui a fait le récit de ses débuts dans la profession, en sortant d'une école d'infirmières à Lille. C'était en 1948, elle avait vingt ans. Nous, Odile Maillard et moi-même, éducatrices retraitées de la PJJ, sommes allées la rencontrer chez elle, à Versailles, en février 2017, pour recueillir son témoignage oral, dont le texte qui suit est fortement inspiré. Aujourd'hui, retraitée depuis 1987, elle a 90 ans. Elle se souvient comme au premier jour de son passage, pendant
3. Koeppel Béatrice, Marguerite B. Une jeune fille en maison de correction, Paris, Hachette, 1987.

4. On peut juste regretter l'absence de quelques notes de bas de pages supplémentaires qui auraient complété les références bibliographiques. 
presque deux ans, dans ce " couvent à sabots ", qui est une façon elliptique, imagée, de nommer l'institution pour filles tenue par des religieuses, où elle fut affectée dès sa sortie d'école pour remplacer une infirmière partie en longue maladie. Si elle ne précise à aucun moment ni le lieu ni le nom exact de cette institution, ne donnant que le département de son implantation, le Nord, c'est tout à fait volontairement. Voilà ce qu'elle en dit dès la première phrase de son introduction: "Au début du siècle dernier, dans le Nord, les "couvents à sabots" étaient des "maisons de redressement" tenues par des sœurs "spécialisées" où on enfermait des filles mineures souvent coupables de faits graves. »

Elle explique aujourd'hui qu' elle ne s'est pas autorisée à nommer expressément de quel couvent elle parle, soit parce que ces maisons avaient une terrible réputation, soit par crainte d'encourir le courroux de la congrégation en dévoilant son nom et ce qui se passait derrière les murs. Nous savons aujourd'hui qu'il s'agit de la congrégation Notre-Dame de Charité du Bon Pasteur d'Angers, dont la maison mère a effectivement été fondée à Angers au milieu du XIX $x^{\mathrm{e}}$ siècle, et dont la fondatrice a essaimé partout en France, tout particulièrement dans le Nord, à Saint-Omer, Lille, Loos, Arras, Marcq-en-Barœul, Amiens. D’après elle, tous furent désignés par la population comme "les couvents à sabots ». Aujourd'hui, Christiane Pécriaux révèle que celui où elle fut infirmière, de 20 à 22 ans, se situait à Loos et qu'il n'en reste plus rien, les bâtiments ayant été entièrement rasés. Elle donne aujourd'hui des précisions qui seront utiles au lecteur pour évaluer, d'une part, dans quel cadre ce livre a été conçu, et d'autre part, la façon dont vivaient les fillettes et les jeunes filles, ainsi que les personnels, surtout des religieuses et quelques laïques, en ce lieu de clôture. Lorsqu'elle y est arrivée, elle habitait chez ses parents, à Lille, et faisait chaque jour une demi-heure de trajet à bicyclette pour se rendre à son travail, matin et soir. Elle dit d'elle-même que, bien élevée, bonne élève, et surtout bien jeunette, innocente malgré ses études d'infirmière, cette immersion dans un couvent habité de femmes et de 200 jeunes filles fut une terrible initiation qui a marqué sa vie entière. Elle aurait souhaité alors travailler dans les hôpitaux, qu'elle connaissait pour y avoir fait des stages au cours de sa formation: les grands blessés, les gros pansements, elle connaissait. Mais ce travail à faire pour tant de filles, dont les plus anciennes avaient pratiquement son âge et connaissaient de la vie bien plus qu'elle-même, quelle expérience! À sa sortie de l'école, sa formatrice lui avait dit: «Ça sera dur, mais vous y arriverez! » Et en effet, la jeune Christiane 
a tenu bon, même au-delà de ce qu'elle avait envisagé au départ, car les trois mois dont on lui avait parlé sont devenus deux années. Toutefois, c'est elle qui a décidé de partir, et quand elle l'a annoncé à la Mère supérieure, celle-ci lui a ordonné de ne pas prévenir les filles de son départ. Ce fut un crève-cœur, ditelle, de les quitter, mais son oppression ressentie à vivre là devenait dangereuse pour son équilibre: personne à qui parler, impression d'être totalement retirée du monde, certitude que personne ne pouvait comprendre. C'est de cette solitude dont elle a le plus souffert. À ses frères et sœurs cadets, elle ne pouvait pas raconter ce qu'elle vivait, pas plus qu'à ses amies. Elle n'écrit alors rien, mais tout est imprimé dans sa mémoire. Et puis, c'est grâce au cahier qu'elle avait emporté dans ses bagages en partant, le " cahier de pesées ", qu'elle put reconstituer toutes les histoires des filles rencontrées au fil des jours. Elle constitua des fiches qui lui servirent de matière pour la rédaction de son livre, écrit bien plus tard, lorsqu'elle fut en retraite. Ce sont ces portraits fugitifs, ces flashes sur telle ou telle de «ses » filles, qui constituent la matière grise de son ouvrage. Sur le dossier médical, elle devait noter l'identité de chaque enfant, les noms et prénoms de son réel état civil, pour les examens à l'extérieur et les visites de contrôle. Et dans un coin en haut de la page, au crayon rouge, elle notait le prénom qui lui avait été attribué à son arrivée, prénom d'emprunt qui suivrait la pensionnaire pendant tout le temps de son placement, le seul que l'infirmière devait prononcer. L'injonction était formelle: "Il vous est interdit de les appeler autrement! » Aujourd'hui, elle estime que tout était fait pour que ces enfants perdent l'identité avec laquelle elles arrivaient. À leur entrée, elles étaient déshabillées pour revêtir un uniforme, vêtements tricotés de coton écru, mais assorti d'un « joli tablier bleu ciel ", précise-t-elle. Donnons les détails qu'elle fournit, qui seront utiles au lecteur souhaitant comprendre le cadre dans lequel évoluait toute cette jeunesse enfermée. À son recrutement, la Mère supérieure lui avait dit que les deux cents pensionnaires dont elle aurait la charge étaient divisées en deux sections: d'une part, le "redressement " ou " grande classe ", regroupant 120 filles, de 11 à 21 ans, la plupart coupables de faits plus ou moins graves; il y régnait la violence, c'était pour elle comme " une cocotte-minute prête à exploser »; d'autre part, la " préservation » ou " petite classe », pour 80 enfants de 5 à 21 ans, retirées d'un milieu dangereux (alcoolisme, maltraitance, inceste).

Celles de la " petite classe " n'étaient pas astreintes à l'uniforme, elles allaient à l'école à l'extérieur, jusqu'au certificat d'études pour celles qui en étaient 
capables, et étaient ensuite " placées » dans la ville; par contre, celles qui n’obtenaient pas l'examen restaient là et travaillaient dans un atelier, de couture le plus souvent, sombre, pas ou peu chauffé, surveillées par une religieuse juchée sur une estrade, dans un silence mortel. Pour celles de la "grande classe ", Christiane Pécriaux n'avait pas beaucoup d'informations sur leur vécu, d'autant qu'elle n'avait pas le droit d'aller les voir dans leurs ateliers, de couture également, ou buanderie, etc. L'interdiction était assortie d'une menace latente: «On ne sait jamais ce qui pourrait se passer!» Tout ce qu'elle pouvait glaner se récoltait lors de leurs visites à l'infirmerie, aménagées par les sœurs à des heures différentes de façon à ce qu'elles ne se croisent pas. L'infirmière, qui avait aussi reçu l'ordre de ne pas les interroger lors de ces visites médicales, dit aujourd'hui que, si elle a obéi au début parce que trop jeune pour se rebeller, elle a fini par passer outre l'interdiction de savoir quoi que ce soit: comment les soigner sinon? Pour ces filles-là, les seules sorties se faisaient avec l'infirmière, par deux ou trois, pour des visites médicales aux laboratoires, ou chez le dentiste, ou à l'hôpital. Elle dit avoir souvent eu la peur au ventre, craignant qu'elles ne s'échappent, ce qui n'est jamais arrivé. Des pensionnaires pourtant réussissaient quand même à s'enfuir, depuis le fond du jardin, à partir d'un grand mur de trois mètres, hérissé de tessons de bouteille; elle n'a jamais compris comment elles s'y prenaient. Aux retours de fugues - " évasions " disait-on - elles étaient durement sanctionnées. Elles avaient droit à «la pénitence », étaient soumises à des punitions sévères, humiliantes: les cheveux rasés; habillées d'une robe de jute informe, tel un sac de pommes de terre; mises au cachot, c'est-à-dire un cagibi où elles risquaient de voisiner avec des rats; et contraintes à un travail de démolition dehors, brique par brique, quel que soit le temps, d'un bâtiment détruit par un bombardement. Au bout de huit à quinze jours, elles reprenaient la vie « normale » dans le groupe.

Dans son livre-souvenir, Christiane Pécriaux raconte les rencontres dans son infirmerie avec ces filles, en soulignant combien la présence à ses côtés, le mardi, du jeune médecin venant de la ville lui était un réconfort. Dès leur arrivée, les filles étaient examinées entièrement. Le toucher vaginal pratiqué à toutes, même aux petites, faisaient souvent découvrir de gros dégâts. Elles étaient en plus soumises à une prise de sang, à la recherche d'une anémie, d'une grossesse ou de la syphilis. Lors des épidémies pouvant atteindre le groupe entier - il y en eut trois pendant ses mois de service: grippe, scarlatine, 
oreillons -, elle était secondée par une auxiliaire qui lui était également d'une grande aide. Des pages écrites à l'issue de ces fugitives entrevues, il ressort une immense compassion, de la tendresse, de la part de la professionnelle pour ces enfants meurtries. Le pire pour elle fut d'être confrontée à la cruauté de sa position d'impuissance face à des situations dramatiques d'enfants placées là, arrivant détruites, souvent très jeunes. Certaines avaient été prostituées par leurs ascendants. Elle dit: "le drame de ma vie professionnelle ", quand elle évoque la mort d'une enfant de quinze ans, Myriam, arrivant "violée, enceinte et syphilitique ", pour laquelle il n'était absolument pas question d'avortement médicalement pratiqué. C'est pourtant vraisemblablement la raison de sa mort: l'infirmière a deviné que ses camarades, pensant l'aider, s'étaient servies d'une aiguille à tricoter, dont elles étaient pourvues pour constituer leur trousseau. Cette jeune fille décède à l'hôpital où elle est autopsiée - «ça se fait pour toutes les filles qui meurent dans la maison ", lui dit la Mère supérieure. Dix jours plus tard, on l'envoie assister à la mise en bière du corps, qu'elle découvre en pièces détachées, les membres séparées du tronc, qu'elle doit remettre en place. Elle relate aussi l'histoire d'une enfant de cinq ans, Isbergue, destinée à la " petite classe ", arrivant dans un état effroyable: battue, violée, une jambe brisée et le corps plein de brûlures de cigarettes. Ces tragédies, Christiane Pécriaux les a traversées sans qu'aucune information ne traverse l'épais mur de silence que les religieuses maintenaient farouchement. Elle aurait tant aimé que l'une ou l'autre s'adresse à elle, l'infirmière, dans ces circonstances. Mais non, tout ce qu'on lui demandait, c'était de faire son travail sans état d'âme. Quand elle sollicitait le soutien de la Mère supérieure, elle se faisait rabrouer. Les pages de son livre sont remplies de ces prénoms féminins qui ont rempli sa vie et peuplent toujours ses souvenirs. Aujourd'hui, elle dit: « les petites prostituées, je les aimais bien, elles étaient généreuses »; ou bien elle évoque: "Georgette, ma préférée, infanticide à 13 ans ". Elle ajoute: "J'ai été trop obéissante, j'aurais pu leur apporter plus; je les aimais bien ces filles."

Elle regrette particulièrement le manque de concertation, de communication a minima avec les religieuses, même si elle dit, au détour d'une phrase, que certaines pouvaient se montrer humaines. Par exemple, elle se souvient que la sœur qui s'occupait des grandes pouvait se dévoiler, se montrant émue, sincèrement peinée, lorsqu'une nouvelle petite arrivait syphilitique. Un bon souvenir qu'elle garde, c'est qu'il y avait de la bonne nourriture au Bon Pasteur: elle y 
mangeait mieux que dans sa famille, en cette période d'après-guerre toujours astreinte au rationnement. Les repas étaient pour elle l'occasion de rencontrer et de parler un peu avec son auxiliaire d'une part - une ancienne pensionnaire vraisemblablement -, et d'autre part les quelques autres laïques travaillant là - une secrétaire et sa sœur innocente, une buandière, une piqurière (pour la réfection des tissus, à l'atelier des pensionnaires aux doigts très abîmés par les aiguilles) et elle, l'infirmière. C'est tout! Du côté des religieuses, elles n'étaient guère plus nombreuses: six ou sept entourant la Mère supérieure. Pas de psychologue, pas de psychiatre, pour compléter l'encadrement de cette multitude d'enfants abîmées par la vie dehors, représentantes de la misère humaine, victimes traitées comme un troupeau. Lorsqu'elle a fait le choix de partir, Christiane Pécriaux a été affectée dans un hôpital, où elle a enfin pu trouver son bonheur dans ce métier dont elle avait la vocation. L'encadrement dans les années 1950 était encore très souvent tenu par des religieuses. Elle a été ensuite formatrice, cadre en milieu hospitalier, alors nommée "surveillante». Une pointe d'amertume transperce son propos: "Quand je suis sortie de là, je n'avais plus confiance dans la parole des gens. " L'écriture de son livre a certainement représenté une catharsis pour elle qui a clos notre entretien par ces mots: "Je suis devenue adulte très vite: la guerre, et puis ça! » Pour conclure, gageons que le lecteur, peut-être a priori effaré par la description des méthodes de traitement des problèmes féminins de l'époque, de ce qu'on appelait « redressement » en guise de rééducation, saura aussi prendre plaisir, au fil de ces belles pages, à découvrir la force d'amour qui s'en dégage entre ces filles enfermées et leur infirmière, qu'elles appelaient «Moiselle », lui confiant: «Vous, Moiselle, on vous aime bien. »

Claire Dumas

\section{Sauvé d'Auschwitz par l'Assistance publique \\ Charles Waserscztajn \\ 2016 \\ Orléans, Éditions du CERCIL, 184 p., ISBN : 9782954090337, préface de Serge Klarsfeld.}

Le récit de Charles Waserscztajn est remarquable tout d'abord par l'événement absurde qui l'a déclenché. Contrairement à ce que l'on pourrait croire et à ce que laisserait sous-entendre la préface de Serge Klarsfeld, il ne démarre pas à 